\title{
AR-MAPS OBTAINED FROM CELL-LIKE MAPS
}

\author{
GEORGE KOZLOWSKI, JAN VAN MILL AND JOHN J. WALSH
}

\begin{abstract}
The recent solution by J. van Mill of a problem of Borsuk involves using a convexification procedure in order to produce a map $f$ from the Hilbert cube $Q$ to a non-AR $X$ so that each point-inverse $f^{-1}(x)$ is a Hilbert cube. A different method of obtaining AR-maps from cell-like maps is described and is used to show that if there is a dimension raising cell-like map, then there is an integer $n$ and a map $f$ from $Q$ to a non-AR $X$ so that each point-inverse $f^{-1}(x)$ is an $n$-cell or a point.
\end{abstract}

Introduction. As is well known, Taylor's Example [5] can be used to construct a cell-like map $f: Q \rightarrow X$ which is not a shape equivalence (Keesling [2]). This map has the remarkable property that $\sup \left\{\operatorname{dim} f^{-1}(x): x \in X\right\}<\infty$. Taylor's Example was recently used by van Mill [4] to construct a map $g: Q \rightarrow Y$ onto a non-AR $Y$ so that each point-inverse $g^{-1}(y)$ is an AR. A map which has AR's for pointinverses is called, for convenience, an AR-map.

There is a convexification procedure in [4] which is used to replace a cell-like map with an AR-map but this procedure does not yield control on the dimension of the point-inverses of the AR-map. We present a method for obtaining an AR-map from a cell-like map which, for maps with finite dimensional domains, produces an AR-map with finite dimensional point-inverses. As an application, we prove that if there is a cell-like dimension raising map, then there is an AR-map $f$ from $Q$ onto a non-AR $X$ so that $\sup \left\{\operatorname{dim} f^{-1}(x): x \in X\right\}<\infty$. Since it is unknown whether there is a cell-like dimension raising map we have not constructed an AR-map as above. However, we identify a type of "convexification" procedure which would suffice to construct such an example from the Taylor Example (see §3).

1. Preliminaries. Our terminology is standard. A cell-like map is a proper map with each point-inverse having trivial shape. The Hilbert cube is denoted by both $Q$ and $I^{\infty}$. If $f: X \rightarrow Y$ is onto, then the mapping cylinder $M(f)$ of $f$ is the space which is obtained from $X \times[-1,1]$ by identifying the set $f^{-1}(y) \times\{-1\}$ to a point for $y \in Y$ and the double mapping cylinder $\operatorname{DM}(f)$ of $f$ is the space which is obtained from $X \times[-1,1]$ by identifying each of the sets $f^{-1}(y) \times\{-1\}$ and $f^{-1}(y) \times\{1\}$ to points for $y \in Y$. The natural collapse to the base from $M(f)$ to $Y$ (resp., $D M(f)$ to $Y$ ) is denoted $r(f)$ (resp., $\operatorname{Dr}(f)$ ). A proper map $f: X \rightarrow Y$ is called a hereditary shape equivalence (Kozlowski [3]) if $\left.f\right|_{f^{-1}(A)}: f^{-1}(A) \rightarrow A$ is a shape equivalence for

Received by the editors April 15, 1980.

1980 Mathematics Subject Classification. Primary 54C55; Secondary 54B15, 54F45.

Key words and phrases. Cell-like, absolute retract, hereditary shape, equivalence, dimension. 
each closed subset $A \subset Y$. Absolute retracts and absolute neighborhood retracts are compact.

Our construction heavily relies on the following results due to Kozlowski [3]. Parts (b) and (c) are proved in [3] and part (a) follows from techniques of proof therein; we have included a proof of (a) in the Appendix.

1.1. Theorem. Let $X$ be compact and let $f: X \rightarrow Y$ be a cell-like map. Then

(a) $f$ is a hereditary shape equivalence if and only if $\operatorname{Dr}(f): \operatorname{DM}(f) \rightarrow Y$ is a hereditary shape equivalence;

(b) if $X$ is an AR (ANR), then $Y$ is an AR (ANR) if and only if $f$ is a hereditary shape equivalence;

(c) if $Z \subset X$ is a closed subset and contains all the nondegenerate point-inverses of $f$, then $f$ is a hereditary shape equivalence if and only if $\left.f\right|_{z}$ is a hereditary shape equivalence.

2. The construction. We now present our main result.

2.1. Theorem. Let $X$ be compact and let $f: X \rightarrow Y$ be cell-like. Then there is a compact space $Z$ containing $Y$ and an open retraction $s: Z \rightarrow Y$ so that

(1) $s$ is an AR-map;

(2) $s$ is a hereditary shape equivalence if and only if $f$ is a hereditary shape equivalence;

(3) $\sup \left\{\operatorname{dim} s^{-1}(y): y \in Y\right\}<2 \cdot \operatorname{dim} X+1$.

Proof. We choose an embedding $X \subset I^{n}$ where $n>2 \cdot \operatorname{dim} X+1$ ( $n$ can be infinite) and let $\Gamma(f) \subseteq I^{n} \times Y$ be the graph of $f$. The space $Z$ is the quotient space obtained from $I^{n} \times Y$ identifying each set $f^{-1}(y) \times\{y\}$ to a point for $y \in Y$ and the retraction $s: Z \rightarrow Y$ is the mapping induced by the projection of $I^{n} \times Y$ to $Y$. Since each $s^{-1}(y)$ is an $n$-cell or Hilbert cube with a single cell-like set $\left(f^{-1}(y) \times\right.$ $\{y\})$ identified to a point, $s$ is an AR-map. The map $s$ satisfies condition (3) and is easily seen to be open.

In order to establish the theorem it suffices to consider the commutative diagram

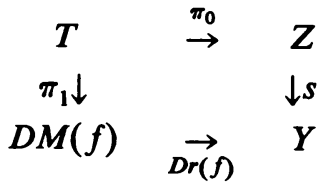

where $T$ is the quotient space obtained from the disjoint union $\left(I^{n} \times Y\right) \cup M(f)$ by identifying $(x, f(x))$ and $(x, 1)$ for each $(x, f(x)) \in \Gamma(f)$, where $\pi_{0} \mid I^{n} \times Y-$ $\Gamma(f)$ is the identity and $\pi_{0} \mid M(f)$ is the collapse to the base, and where $\pi_{1} \mid M(f)-X$ $\times\{1\}$ is the identity and $\pi_{1} \mid I^{n} \times Y$ is the projection to $Y$. Part (c) of Theorem 1.1 can be used to verify that both $\pi_{0}$ and $\pi_{1}$ are hereditary shape equivalences. An easy diagram "chase" establishes that $s$ is a hereditary shape equivalence iff $\operatorname{Dr}(f)$ is a hereditary shape equivalence and an appeal to part (a) of Theorem 1.1 completes the proof. 
2.2. Remark. It is an easy matter to choose the embedding $X \subset I^{n}(n>5)$ so that each point-inverse $s^{-1}(y)$ is an $n$-cell (or a Hilbert cube for $X$ infinite dimensional); specifically, for $n$ finite choose a 1-LCC embedding of $X$ into the interior of $I^{n}$ and for $n$ infinite choose a $Z$-embedding of $X$. In fact, with these choices the map $s$ is completely regular in the sense of Dyer-Hamstrom [1]. A consequence of this is that the AR-map $F$ in the next corollary can be chosen so that each point-inverse $F^{-1}(s)$ is a point or an $n$-cell.

2.3. Corollary. If there is a cell-like dimension raising map, then there is an AR-map $F: Q \rightarrow S$ onto a non-AR $S$ so that $\sup \left\{\operatorname{dim} F^{-1}(s): s \in S\right\}<\infty$.

Proof. It is known (cf. Kozlowski [3]) that if there is a cell-like dimension raising map, say $f: X \rightarrow Y$, then $f$ is not a hereditary shape equivalence. By Theorem 2.1, there is a compact space $Z$ containing $Y$ and an AR retraction $s: Z \rightarrow Y$ so that $s$ is not a hereditary shape equivalence and so that $\sup \left\{\operatorname{dim} s^{-1}(y): y \in Y\right\}<\infty$. Let $Z \subset Q$ and let $S=Q \cup_{s} Y$ be the adjunction space. The induced quotient map $F: Q \rightarrow S$ is not a hereditary shape equivalence since $s$ is not. Consequently, by part (b) of Theorem 1.1, $S$ is not an AR.

2.4. Remark. Starting with a cell-like mapping $f: Q \rightarrow Y$ which is not a hereditary shape equivalence, the argument in the proof of Corollary 2.3 yields an AR-map $F: Q \rightarrow Y$ onto a non-AR $Y$. This map and the space $Y$ are similar to those in van Mill [4].

3. A question. The Taylor Example yields a cell-like map $f: X \rightarrow Y$ between infinite dimensional compacta which is not a hereditary shape equivalence with $\sup \left\{\operatorname{dim} f^{-1}(y): y \in Y\right\}<\infty$. If the following question has an affirmative answer, then Corollary 2.3 holds without the assumption that there exists a cell-like dimension raising map. We have answered the question for finite dimensional $X$.

3.1. Question. Let $f: X \rightarrow Y$ be a cell-like map between compacta. Does there exist a compact space $W$ containing $X$ and an extension $F: W \rightarrow Y$ of $f$ such that

(1) $F$ is an AR-map;

(2) $F$ is a hereditary shape equivalence;

(3) if $\sup \left\{\operatorname{dim} f^{-1}(y): y \in Y\right\}<\infty$, then $\sup \left\{\operatorname{dim} F^{-1}(y): y \in Y\right\}<\infty$.

Starting with such a map $F: W \rightarrow Y$, if $Z$ is the quotient space obtained from $W$ by identifying each set $f^{-1}(y)$ to a point for $y \in Y$ and if $s: Z \rightarrow Y$ is the map induced by $F$, then the proof of Theorem 2.1 applies with $W$ taking the place of $I^{n} \times Y$ and shows that the AR-map $s$ is a hereditary shape equivalence if and only if $f$ is a hereditary shape equivalence.

Appendix. Let $f: X^{\prime} \rightarrow X$ be a map. For a subset $A \subset X$, the inverse set $f^{-1}(A)$ is denoted $A^{\prime}$ and the mapping cylinder of the restriction of $f$ to $A^{\prime}$ is considered to be a subset of the mapping cylinder $M(f)$ and is denoted $M(f, A)$. Let $Z \subset X$ be a closed subset and let $\alpha: Z \rightarrow Y$ be a surjective map. The adjunction space of $\alpha$, denoted $X \cup_{\alpha} Y$ is the quotient space obtained from $X$ by identifying each point-inverse $\alpha^{-1}(y)$ to a point; the induced quotient map is denoted $\pi_{\alpha}: X \rightarrow X$ $\cup_{\alpha} Y$. The space $Y$ is "naturally" identified with a subspace of $X \cup_{\alpha} Y$. 
Proposition. Let $f: X^{\prime} \rightarrow X$ be a cell-like map between compact spaces. Then $f$ is a hereditary shape equivalence if and only if $\operatorname{Dr}(f): D M(f) \rightarrow X$ is a hereditary shape equivalence.

Proof. Suppose that $f$ is a hereditary shape equivalence and consider $X^{\prime}$ to be a subset of an ANR $W$. Let $\alpha: X^{\prime} \times\{0,1\} \rightarrow X \times\{0,1\}$ be defined by $\alpha(x, i)=$ $(f(x), i)$ for $i=0,1$. The map $\alpha$ is a hereditary shape equivalence and, therefore, parts (b) and (c) of Theorem 1.1 combine to imply that $(W \times I) \cup_{\alpha}(X \times\{0,1\})$ is an ANR. For the same reasons, $W \cup_{f} X$ is an ANR. The composition of the projection of $W \times I$ to $W$ and the quotient map $\pi_{f}: W \rightarrow W \cup_{f} X$ induces a map $q:(W \times I) \cup_{\alpha}(X \times\{0,1\}) \rightarrow W \cup_{f} X$. Since $q$ is a cell-like map between ANR's, part (b) of Theorem 1.1 implies that $q$ is a hereditary shape equivalence. The restriction of $q$ to $q^{-1}(X)$ is the map $\operatorname{Dr}(f): D M(f) \rightarrow X$ and, hence, the latter map is a hereditary shape equivalence.

Using basic results from [3], in order to show that $f$ is a hereditary shape equivalence, it suffices to show that, for any pair of closed subsets $A \subset B$ of $X$, any map $h: M(f, A) \cup B^{\prime} \rightarrow P$ into an ANR $P$ extends to a map $H: M(f, B) \rightarrow P$. Since $f$ is a cell-like map, there is a closed cover $A_{0} \supset A, A_{1}, \ldots, A_{n}$ of $B$ and maps $h_{i}: M\left(f, A_{i}\right) \rightarrow P$ with $h_{0}=h$ on $M(f, A)$ and, for $i \geqslant 1, h_{i}=h$ on $A_{i}^{\prime}$.

Let $H_{0}=h_{0}$ and inductively assume that $H_{i}: M\left(f, A_{0} \cup \cdots \cup A_{i}\right) \rightarrow P$ agrees with $h$ on $M(f, A) \cup\left(A_{1}^{\prime} \cup \cdots \cup A_{i}^{\prime}\right)$. Let $D=\left(A_{0} \cup \cdots \cup A_{i}\right) \cap A_{i+1}$ and let $g$ be the restriction of $f$ to $D^{\prime}$. Define a map $\beta: D M(g) \rightarrow P$ by using the restriction of $H_{i}$ on one copy of the mapping cylinder and the restriction of $h_{i+1}$ on the other copy. By assumption the map $\operatorname{Dr}(g): \operatorname{DM}(g) \rightarrow D$ is a shape equivalence and, therefore, $\beta$ extends over the mapping cylinder of $\operatorname{Dr}(g)$. This extension yields a homotopy rel. $D^{\prime}$ between the restrictions of $H_{i}$ and $h_{i+1}$ to $M(f, D)$. Since $h_{i+1}$ extends over $M\left(f, A_{i+1}\right)$, the map $H_{i}$ also extends producing the map $H_{i+1}$. A more detailed argument can be found in [3].

\section{REFERENCES}

1. E. Dyer and M. E. Hamstrom, Completely regular mappings, Fund. Math. 45 (1957), 103-118.

2. J. Keesling, A non-movable trivial-shape decomposition of the Hilbert cube, Bull. Acad. Polon. Sci. Sér. Sci. Math. Astronom. Phys. 23 (1975), 997-998.

3. G. Kozlowski, Images of ANR's, Trans. Amer. Math. Soc. (to appear).

4. J. van Mill, A counterexample to a question of Borsuk on decomposition of absolute retracts (to appear).

5. L. Taylor, A counterexample in shape theory, Bull. Amer. Math. Soc. 81 (1975), 629-632.

Department of Mathematics, Auburn University, Auburn, Alabama 86830

Department of Mathematics, Louisiana State University, Baton Rouge, Louisiana 70803

Department of Mathematics, University of Tennessee, Knoxville, Tennessee 37916 